\title{
Aortic Dissection with Rare Anatomical Aortic Arch Variation Depicted by Computed Tomography Angiography
}

\author{
Xin Wang, MD*, ${ }^{1}$ Bofu Liu, MD*, ${ }^{2}$ Haifang Yu, $\mathrm{MD}^{2}$ \\ ${ }^{1}$ Department of Thoracic Surgery, Sichuan Cancer Hospital \& Institute, Sichuan Cancer Center, School of Medicine, University of \\ Electronic Science and Technology of China, Chengdu, China; ${ }^{2}$ Department of Emergency Medicine, West China Hospital, Sichuan \\ University, Chengdu, China
}

\section{ABSTRACT}

Aortic dissection (AD) is a life-threatening disease, and endovascular repair by stent graft is an effective treatment. Surgery often fails for a variety of reasons, such as aortic arch variation. We present the case of a 27-yearold female with aortic dissection with a rare anatomical aortic arch variation caused by chest trauma. This patient recovered well after endovascular repair. This case report demonstrates endovascular repair can be applied to aortic dissection patients with rare anatomical aortic arch variation.

\section{CASE REPORT}

A 27-year-old female was transferred to our hospital four hours after a traffic accident with chest trauma. Multiplanar reconstruction and volume rendered CT revealed a rare aortic arch variation with two branches - a common trunk of bilateral common carotid arteries and a common trunk of bilateral subclavian arteries (Figures $1 \mathrm{~A}$ and 1B). CT angiography also discovered Stanford B-type aortic dissection from the aortic arch to the thoracic aorta, however the branches of the aortic arch were not involved (Figure 1C). Angiography revealed the intimal tear of the aorta was located $2.5 \mathrm{~cm}$ distal to the common trunk of bilateral subclavian arteries, and patient then underwent endovascular repair by a stent graft (Figure 1D). The postoperative status was uneventful, and the patient recovered well.

\section{DISCUSSION}

Aortic dissection (AD) is a life-threatening disease with an incidence of approximately three per 100,000/

Received Fanuary 18, 2021; received in revised form March 1, 2021; accepted March 3, 2021.

*Xin Wang and Bofu Liu contributed equally.

Correspondence: Pro Haifang Yu, MD, Department of Emergency Medicine, West China Hospital, Sichuan University, 37 Guoxue Road, Chengdu 610041, Sichuan, China; +86-28-85422288 (e-mail: yuhaifang@wchscu.cn). year [Mészáros 2000]. Patients usually need to undergo expensive endovascular repair by a stent graft to save lives. Variations in the branching pattern of the aortic arch are multiple. Much literature has reported the incidence of variations of aortic arch from $13.3 \%$ to $26 \%$ [Natsis 2009]. Although the occurrence of aortic branch variability is rare, there are a few of these variabilities that may directly lead to clinical misdiagnosis or surgical failure [Qiu 2019]. Therefore, it is imperative to summarize the treatment experience of $\mathrm{AD}$ patients with aortic arch variation.

In our case, we found a rare case of aortic arch variation with an incidence of only $0.16 \%$. This variation is also named avian aortic arch (avian form); it is defined by its

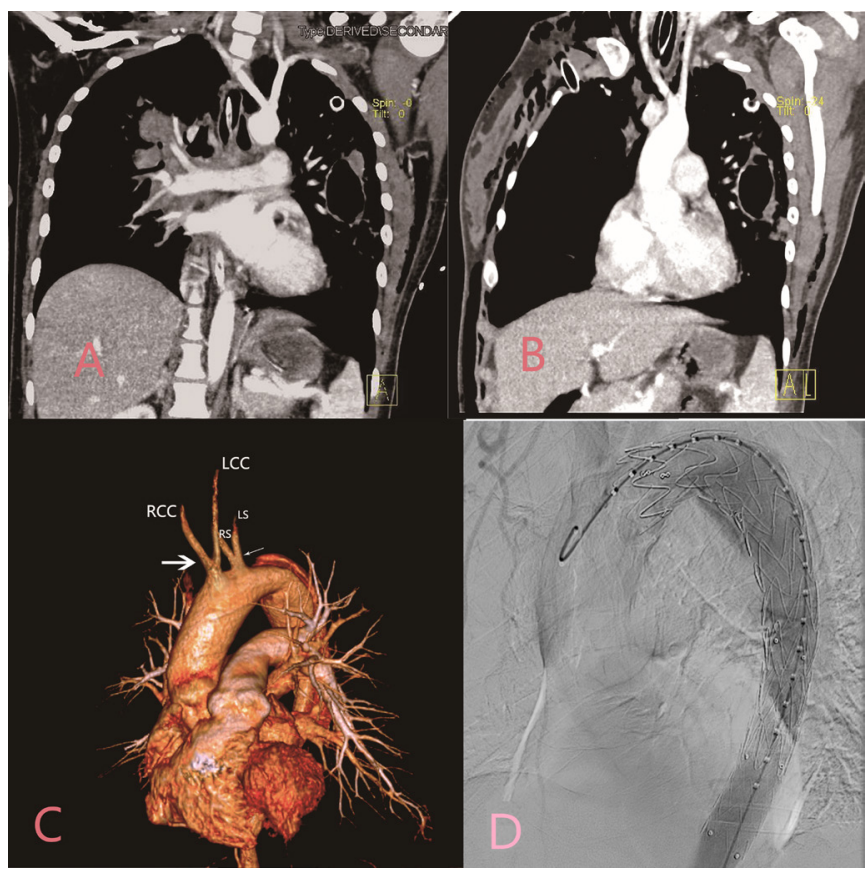

A, A common trunk for both subclavian arteries; B, A common trunk giving origin to the common carotid arteries; $C$, Volume rendered $C T$ image. Thin arrow: common trunk for bilateral subclavian arteries; D, Endovascular repair by a stent graft. Thick arrow: common trunk for bilateral common carotid arteries. RCC, right common carotid artery; LCC, left common carotid artery; RS, right subclavian artery; LS, left subclavian artery 
common origins of bilateral common carotid and subclavian arteries. It is a rare variation in the branching pattern of aortic arch with few cases reported and found by angiography and autopsy [Ergun 2014]. In terms of treatment, the patient underwent endovascular repair by a stent graft and successfully was discharged. However, 3D printed patientspecific aortic stent graft would be preferred if aortic dissection involved an uncommon aortic arch [Sun 2020]. This provides a reference for our treatment decisions for $\mathrm{AD}$ patients with aortic arch variation.

\section{ACKNOWLEDGEMENTS}

This work was financially supported by grants from the Science Foundation of Science and Technology Department of Sichuan (No. 2018SZ0390).

\section{REFERENCES}

Ergun O, Tatar IG, Durmaz HA. 2014. A case of a rare aortic arch variation: the avian form. JBR-BTR. 97(6):349-50.

Mészáros I, Mórocz J, Szlávi J. 2000. Epidemiology and clinicopathology of aortic dissection. Chest. 117:1271 ר-8.

Natsis KI, Tsitouridis IA, Didagelos MV. 2009. Anatomical variations in the branches of the human aortic arch in 633 angiographies: clinical significance and literature review. Surgical and radiologic anatomy: SRA. 31(5):319-323.

Qiu Y, Wu X, Zhuang Z. 2019. Anatomical variations of the aortic arch branches in a sample of Chinese cadavers: embryological basis and literature review. Interact CardioVasc Thorac Surg. 28(4):622-628.

Sun Z. 2020. Clinical Applications of Patient-Specific 3D Printed Models in Cardiovascular Disease: Current Status and Future Directions. Biomolecules. Nov 20;10(11):1577. 